\title{
Pólipos endometriales: Actualización en diagnóstico y tratamiento
}

\author{
Andrés Vigueras S. ${ }^{1}$, Juan Raúl Escalona M. ${ }^{1}$ \\ ${ }^{1}$ Departamento de Ginecología y Obstetricia, Facultad de Medicina, Universidad de Concepción. Servicio de Obstetricia \\ y Ginecología, Hospital Las Higueras, Talcahuano. Chile.
}

\section{RESUMEN}

Antecedentes: Los pólipos endometriales son protrusiones nodulares benignas de la superficie endometrial con características clínico-patológicas propias y de origen desconocido. Su prevalencia es alta, especialmente en pacientes con sangrado uterino anormal, se asocia a infertilidad y a cáncer de endometrio, lo que se traduce en que se trata de una patología muy importante en el quehacer ginecológico habitual. Objetivos: Revisar la patogenia, actualizar y evaluar los métodos diagnósticos, y definir las mejores opciones terapéuticas de esta frecuente patología. Método: Revisión sistemática de la literatura publicada en el tema, mediante búsqueda en base de datos Pub Med. Resultados: La patogenia es aun desconocida, es una patología muy heterogénea y no hay causa única, se han reportado varios hallazgos relacionados con alteraciones genéticas. La ultrasonografía de alta definición, la hidrosonografia y la histeroscopia son el estándar actual en el diagnóstico. Las opciones terapéuticas se extienden desde la observación y seguimiento con imágenes, hasta la histerectomía con biopsia contemporánea, siendo la histeroscopia quirúrgica el método más costo efectivo. Conclusión: La patogenia de los pólipos endometriales se encuentra actualmente en revisión, no hay ninguna teoría que explique la génesis de todos los pólipos. La ultrasonografía con contraste y la histeroscopia constituyen el estándar en el diagnóstico. En el tratamiento, la indicación es la cirugía histeroscopica y en especial la resección electroquirúrgica, que permite una extracción completa del pólipo bajo visión directa, con enfoque diagnóstico y terapéutico, con riesgos bajos y recurrencia mínima.

\section{PALABRAS CLAVE: Pólipo endometrial, histeroscopia, resectoscopia}

\section{SUMMARY}

Background: Endometrial polyps are benign nodular protrusions of the endometrial surface with clinical and pathological features of unknown origin. Its prevalence is high, especially in patients with abnormal uterine bleeding, it is associated with infertility and endometrial cancer, what constitutes a common and important disease. Aims: To review the pathogenesis, to update and evaluate diagnostic methods, and to define the best treatment options for this common condition. Methods: A systematic review of the published literature on the subject by searching PubMed database. Results: The pathogenesis is still unknown, it is a very heterogeneous disease and there is no single cause, there have been several findings related to genetic alterations. High definition ultrasonography, the hidrosonography and hysteroscopy are the current standard in diagnosis. Therapeutic options range from observation and follow up with images to the hysterectomy using contemporary biopsy, surgical hysteroscopy being the most cost effective method. Conclusion: The pathogenesis of endometrial polyps are currently under review, there is no theory to explain the genesis of all polyps. Contrast ultrasonography and hysteroscopy are standard in the diagnosis. In the treatment, the 
indication is histeroscopy surgery and especially electrosurgical resection, which allows complete removal of polyps, diagnostic and therapeutic approach, with low risk and low recurrence.

\section{Key Words: Endometrial polyp, histeroscopy, resectoscopy}

\section{INTRODUCCIÓN}

Según definición del tratado de clasificación histología de tumores de la Organización Mundial de la Salud, un pólipo endometrial se define como una protrusión nodular benigna sobre la superficie endometrial constituido por glándulas, estroma y vasos sanguíneos característicos. Microscópicamente presenta un estroma fibroso denso al menos focalmente y vasos sanguíneos largos de paredes musculares gruesas. Lo anterior, sumado a la presencia de glándulas irregulares, con dilataciones quísticas, cubiertas por epitelio superficial, serán características claves en el diagnóstico diferencial con otras enfermedades del endometrio (1) (Figura 1). En la pre-menopausia se ha descrito la disposición paralela del eje mayor glandular con la superficie epitelial como característica histológica propia, presente en el $80 \%$ de los casos (2).

Los diagnósticos diferenciales principales son (1): 1. Hiperplasia endometrial. 2. Carcinoma endometrial polipoideo. 3. Adenofibroma. 4. Adenosarcoma. 5. Tumor Mülleriano mixto maligno. 6. Adenomioma polipoideo atípico. De ahí que es necesario precisar la etiología de la estructura polipoidea para ofrecer el tratamiento adecuado.

El objetivo de esta comunicación es revisar la patogenia, actualizar y evaluar los métodos diagnósticos y definir las mejores opciones terapéuticas de esta frecuente patología ginecológica.

\section{PATOGENIA}

Su génesis es desconocida. Las hipótesis propuestas en la literatura son (1): 1. Origen monoclonal: constituyen proliferaciones monoclonales benignas del mesénquima. 2. Anomalías cariotípicas: se han documentado anomalías en clusters de cromosomas 6 (6p21) y 12 (12q15), que alterarían el proceso proliferativo determinando un sobre crecimiento endometrial $(3,4)$. 3 . Aumento de metaloproteinasas de matriz y citokinas: se ha demostrado un aumento al comparar con controles no solo en pólipos endometriales, sino también en múltiples enfermedades uterinas benignas (5). No existe claridad en si el fenómeno obedece a la causa o como consecuencia de la enfermedad. 4. Sobreexpresión de proteína P63: proteína marcadora y reguladora de las células de reserva de la capa basal del endometrio, su expresión estaría aumentada en portadoras de pólipos endometriales, y sería la causa
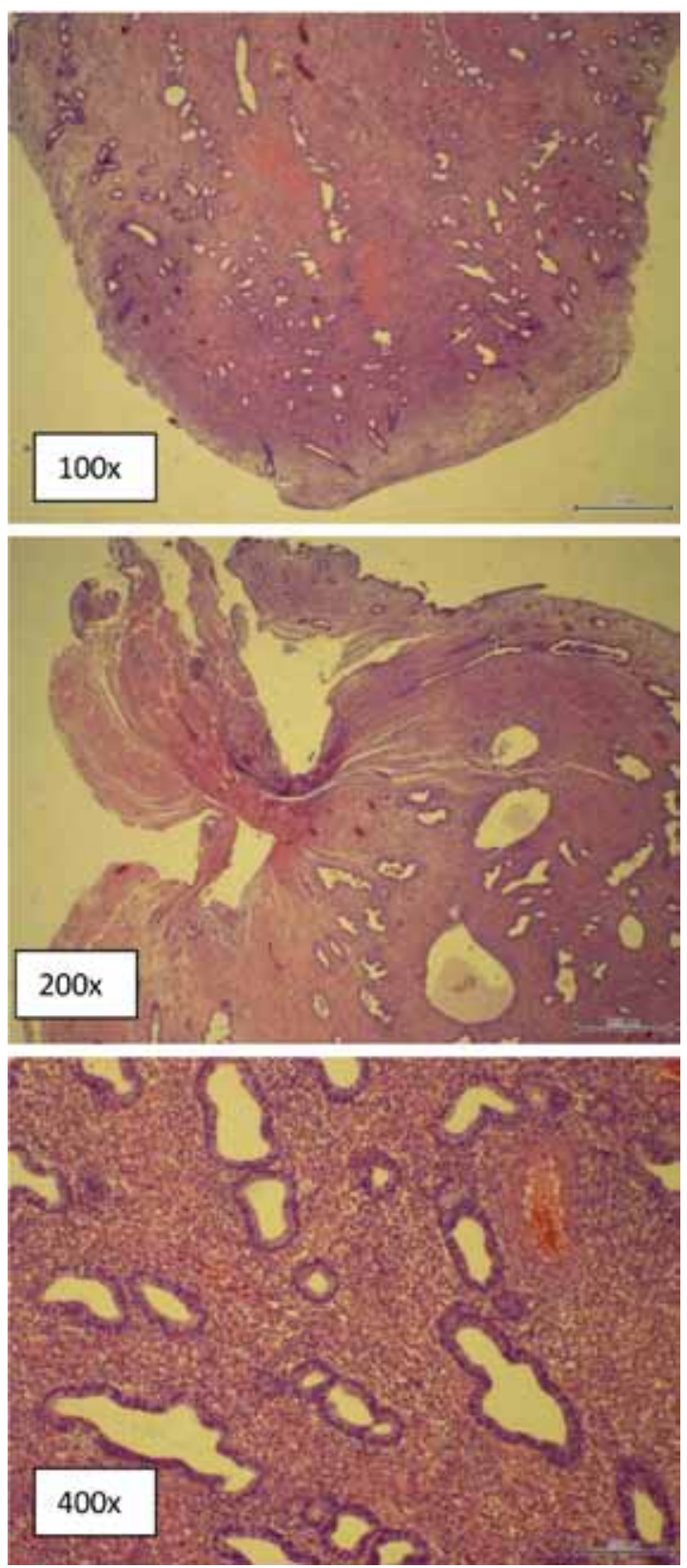

Figura 1. Pólipo endometrial con 3 aumentos diferentes (100x, 200x y 400x). Unidad de Anatomía Patológica, Hospital Las Higueras. 
de la aparición de estas lesiones en la postmenopausia (6). 5. Desbalance niveles estrógenos/progesterona: hormonas reguladoras de proliferación y apoptosis en el endometrio, pareciera ser que ambas contribuyen en la elongación de arterias espirales, estroma y glándulas endometriales dando la apariencia polipoídea a estos crecimientos. Se presentan en sitios con mayor expresión de receptores de estrógeno, disminución de receptores de progesterona o ambos $(7,8)$. Se discute predominio estrogénico y glandular de estos receptores, sobre todo en pacientes post menopáusicas (9). 6. Mutaciones genéticas: genes HMGIC y HMGIY, que determinan un sobreexpresión de la aromatasa endometrial lo que ocasiona una mayor producción de estrógenos locales con la subsecuente sobre estimulación de la proliferación (10).

Dado la heterogeneidad de la patología, el reconocer una causa única resulta improbable. En su etiopatogenia se han descrito factores predisponentes ampliamente estudiados y aceptados: 1 . Hipertensión arterial: alteraría mecanismos apoptóticos celulares, favoreciendo el crecimiento celular, esto en todas las neoplasias hormono-dependientes (11-15). 2. Diabetes mellitus Tipo 2: favorece el crecimiento y alteraciones celulares, mediados por aumento de IGF-1 en estados de hiperinsulinemia (14). 3. Obesidad: determina hiperestrogenismo, que estimula receptores endometriales y favorece crecimiento celular $(16,17)$. 4. Edad (17): factor de riesgo conocido y aceptado, con aumento importante después de los 40 años, con mayor prevalencia entre los 45 a 50 años. 5. Tamoxifeno: Se informa de un 30 a $60 \%$ de prevalencia (1). 6. Estado menopáusico: controversial, se reportan prevalencias superiores en postmenopausicas, sin embargo al estudiarse todas las metrorragias de este grupo es probable que se deba a un sesgo en la selección $(16,17)$. 7. Terapia hormonal: controversial, se ha descrito tanto mayor como menor prevalencia $(18,19)$.

Como factores protectores en su desarrollo, mediante efecto anti-estrogénico a nivel endometrial, se han mencionado los anticonceptivos hormonales (ACO), los progestágenos puros y el dispositivo intrauterino liberador de levonorgestrel $(16,18,20)$. Sin embargo, es necesario estudios metodológicamente adecuados para confirmar estos resultados.

Si bien podrán seguir ciclidad endometrial, es decir que proliferen y descamen como el endometrio normal, esto no siempre sucede y en ocasiones mantienen su estructura inalterable a pesar del ciclo hormonal (21).

Para efectos prácticos, los pólipos endometriales carecen de una clasificación morfológica precisa, sin embargo suelen sub-clasificarse patológicamente de la siguiente forma: 1. Pólipos hiperplásicos: asemejan el aspecto de una hiperplasia difusa del endometrio, pero no existe evidencia que represente la misma entidad. 2. Pólipos atróficos: alineamiento celular columnar o cuboidal bajo, característico en postmenopausia. 3. Pólipo funcional: relativamente infrecuente, sigue ciclicidad endometrial normal (22).

Podrán ser sésiles o pediculados, únicos o múltiples, desde milímetros hasta algunos centímetros, y si bien suelen ser de predominio fúndico, su localización podrá ser en cualquier sitio de la cavidad endometrial. Se ha descrito entre un 24 a $27 \%$ de asociación entre pólipos cervicales y endometriales (23-26), que aumenta con la edad y la presencia de sangrado anormal $(17,18)$. De igual forma, parece existir asociación a otras enfermedades ginecológicas benignas como endometriosis y leiomiomas $(17,18)$. En pacientes con PAP atípico glandular se encontrará un pólipo endometrial en un 3,4 a 5\% (1).

\section{CLÍNICA Y EPIDEMIOLOGÍA}

Su prevalencia fluctúa entre 7,8 a 34,9\% según trabajos clásicos $(6,18,27-29)$. El amplio intervalo esta dado por diferencias en definición, método diagnóstico y población estudiada (17). Gracias a la ultrasonografía de alta definición, la hidrosonografía y la histeroscopia, su frecuencia diagnóstica ha aumentado ostensiblemente (30-32). Es así como en pacientes con sangrado uterino anormal su prevalencia llega a un $10-40 \%(6,27,33,34)$, no superando el $12 \%$ en grupos asintomáticos estudiados por otras causas $(18,35)$. Son más frecuentes entre los 40 y 50 años, con aumento gradual antes y disminución posterior a este grupo etario (28).

La incidencia general es desconocida, considerando que hasta un $30 \%$ podrán ser asintomáticos (34-37). Según reportes recientes, un 56 a $88 \%$ de los pólipos serán sintomáticos (15). El 64-88\% de las premenopáusicas y el $56 \%$ de las postmenopáusicas serán sintomática $(35,38)$.

El síntoma predominante es el sangrado genital, representando un $68 \%$ de los casos, ya sea en la forma de metrorragia postmenopausica, menorragia, sangrado intermenstrual y/o post coital, y obedecerá a congestión estromal intra- pólipo que determina estasis venoso y necrosis apical (35). La sintomatología no tiene relación con su ubicación, número o tamaño (39).

Otras manifestaciones clínicas descritas:

1. Infertilidad: su prevalencia podrá llegar hasta un $32 \%$ según estudios prospectivos diagnosticados por histeroscopia previo a fertilización in vitro (40). Como causa de infertilidad es poco frecuente y obedece generalmente a pólipos mayores a dos centímetros. Estos, según hipótesis propuestas, determinarán una obstrucción mecánica a nivel del ostium alterando la migración espermática (41) y/o generando alteraciones bioquímicas en la implantación y el desarrollo embrionario (4). Solo un 
estudio randomizado controlado ha logrado demostrar asociación causal entre estas dos patologías (42). 2. Sangrado posterior a legrado uterino, una paciente con metrorragia a la que se le realiza un legrado terapéutico y el sangrado persiste luego del procedimiento. 3. Asintomático: diagnóstico incidental en estudio por imágenes de otras patologías ginecológicas.

\section{POTENCIAL ONCOLÓGICO}

El potencial maligno de un pólipo endometrial es conocido, y varía entre un 0,8 a un $8 \%$ según las distintas series publicadas (12,13,43-47). Existe consenso en que el riesgo de cáncer subyacente aumenta con la edad, y el sangrado se ha reportado como un factor importante de riesgo de malignidad en algunas series (41-48). Una revisión sistemática con metaanálisis realizada en 2010 (15) confirma estos resultados, además, ratifica al estado menopáusico y sintomático como los dos predictores más potentes de malignidad al evaluar un pólipo endometrial, con RR de 3,86 y 1,97 respectivamente. Algunos autores han informado que el pólipo asintomático determina un riesgo despreciable de malignidad $(12,49)$.

Otros factores que aumentan el riesgo oncológico, con evidencia inconsistente, son: HTA, diabetes mellitus tipo II, uso de tamoxifeno (15), obesidad, tamaño mayor a $15 \mathrm{~mm}$ y edad mayor a 60 años (50). En el metaanálisis mencionado solo 8 de los 17 estudios informaron el factor tamaño, cuatro de estos mostraron asociación positiva y directa, mientras otros cuatro no encontraron relación significativa (15). Por su parte, la menopausia tardía, determina un mayor tiempo de exposición a estímulo estrogénico, y se ha correlacionado con mayor riesgo de malignidad (13).

Si bien es infrecuente, las hiperplasias atípicas y el cáncer invasor pueden originarse de un pólipo endometrial. Podrá ser origen de un maligno primario si cumple con tres criterios básicos: tumor confinado en el apex, ausencia de lesión en su base, y endometrio adyacente sin alteraciones. Se informa entre un 0 a 1,8\% de carcinoma confinado al pólipo, dependiendo de la población y método diagnostico utilizado (51).

La presencia de atipias intrapólipo determina su potencial premaligno. Este tendrá hasta un $28 \%$ de transformación maligna en su evolución natural y un $42,6 \%$ de carcinoma endometrial concurrente en piezas de histerectomía (52).

\section{TRATAMIENTO}

A la fecha, existe consenso en la literatura sobre la conducta ante un pólipo endometrial, la mayoría de los autores recomienda la extracción rutinaria de todos los sintomáticos $(7,28,45,46,51)$, otros son algo más conservadores $(35,53,54)$.

En el contexto de búsqueda de fertilidad existe acuerdo en su tratamiento quirúrgico, pues está documentado el aumento en tasas de embarazo, que pueden llegar a un 43 a $80 \%$ (55).

De forma general, 4 factores deberán considerarse al planificar el tratamiento (1): 1. Sintomatología. 2. Riesgo o potencial maligno estimado. 3. Búsqueda de fertilidad. 4. Habilidad del cirujano.

A partir de este esquema de evaluación, se proponen 3 opciones de manejo (1).

Conservador no quirúrgico. Considerando al carácter predominantemente benigno de un pólipo y conociendo factores relacionados a malignidad, el tratamiento expectante sin intervención es propuesto como opción terapéutica en aquellos pólipos asintomáticos y menores a $10 \mathrm{~mm}$ (35), dado su alto rango de resolución espontanea a 12 meses de seguimiento $(53,54)$.

Se ha informado que el tamaño podría ser un factor implicado en la regresión o persistencia de un pólipo. En el estudio transversal de Lieng y cols del 2009 (35), se describe un 27\% de regresión a 12 meses de seguimiento en aquellos pólipos menores a 10,7 mm, siendo aquellos mayores de 15,1 $\mathrm{mm}$ los con mayor chance de persistir $(37,54)$. La regresión se ha asociado a episodios de menorragia y algia pelviana aguda transitoria que puede estar dada por la probable expulsión del pólipo.

Otras terapias médicas como los análogos GnRH que causan menopausia artificial, no cuentan con suficiente evidencia para ser recomendado como tratamiento único, y su mayor utilidad está en el manejo preresección histeroscópica, en casos de pólipos de gran volumen, para reducir su tamaño y así permitir un procedimiento técnicamente más simple $(35,56)$.

Conservador quirúrgico. Actualmente, dentro del manejo quirúrgico conservador, la histeroscopia quirúrgica es considerada el gold standard diagnóstico y terapéutico en enfermedades benignas de cavidad endometrial, incluidas el pólipo endometrial (35). Esto ha permitido conocer mejor las características de la enfermedad. Dada su alta efectividad, bajo riesgo y adecuada costo-efectividad, es ampliamente aceptada como estándar en la resección de pólipos endometriales y evaluación de la cavidad endometrial en general. Se informan sensibilidades y especificidades diagnósticas de $89,9 \%$ y $93 \%$ respectivamente, al comparar con ecografía transvaginal, usando como gold standard el diagnóstico histológico (30). Este método permitirá cuantificar los pólipos, analizar vasculatura, localización, tamaño, forma, sitio de implantación, además de la resección completa bajo anestesia (28). 
Dentro de las múltiples ventajas de la histeroscopia se mencionan: 1. Resección completa del pólipo bajo visión directa; la base del pólipo siempre debe ser incluida. 2. Observación y biopsia de cualquier otra lesión o hallazgo sospechoso en el resto de la cavidad endometrial. 3. Baja tasa de complicaciones $(0,28 \%)(57,58,59)$. 4. Considerado un procedimiento diagnóstico y terapéutico. 5 . Menor estadía hospitalaria y un expedito retorno a la actividad normal (57).

Los métodos y técnicas de polipectomía incluyen el uso de resectoscopios monopolares y bipolares (38), morceladores (58) y otros instrumentos (1). El tipo de instrumento dependerá de la disponibilidad, costo y experiencia del cirujano, además del tamaño y localización del tumor (1). En general, para pólipos grandes y sésiles se recomienda la resectoscopia, mientras que para pequeños y pediculados es preferible la tijera o fórceps $(1,59)$.

Al no escindir el miometrio, el riesgo de complicaciones es baja y según el estudio prospectivo más grande, con más de 13.500 procedimientos no superaría el 0,28\%, porcentaje significativamente menor al compararlos con otros procedimientos como miomectomias o lisis de sinequias (59). Por el mismo concepto anatómico, el riesgo de adherencias post operatorias es baja, no reportándose casos post polipectomía histeroscópica (1)

La recurrencia post histeroscopia quirúrgica es entre un $0-15 \%$, dependiendo del método de resección utilizado (57). Evidencia retrospectiva con 9 años revisión y 240 polipectomias histeroscópicas realizadas, informa una recurrencia del 3,7\%, requiriendo nueva histeroscopia un $1,7 \%$ e histerectomía el 0,8\% (57).

En general, la eficacia de la polipectomía histeroscópica en pacientes con metrorragia es descrita entre un 75 a $100 \%(60,61)$. El único estudio prospectivo randomizado controlado, que evalúa la eficacia de la resección histeroscópica en el manejo del pólipo sintomático, es el publicado por Lieng y cols en 2010, sus resultados informan ausencia de beneficios en disminución de volumen menstrual, con una mejora sintomática significativa en el grupo quirúrgico, sobre todo en relación a sangrado intermenstrual (61).

La correlación visual histeroscopica con benignidad o malignidad de la lesión, es baja e imprecisa (50). Los determinantes de esta serán la experiencia del examinador, forma del pólipo y vascularización de superficie $(50,51)$. Por otra parte, lesiones uterinas pequeñas y/o cambios funcionales del endometrio limitarán el rendimiento de la histeroscopia en el diagnóstico de lesiones focales. Además, las neoplasias malignas de endometrio podrán adquirir aspecto polipoideo indistinguible a ojo desnudo de pólipos endometriales benignos. Es por esto que en pacientes en riesgo de malignidad, la resec- toscopia histeroscópica y biopsias de endometrio adyacente son recomendadas (62). Esta permitirá diferenciar un pólipo con transformación maligna de aquel carcinoma endometrial polipoideo, pues mediante una extracción completa y bajo visión, la extracción de su base es regla y será el estudio histológico de esta la que determine el diagnostico final. Visualización y extracción directa se ha reportado efectiva en reducir porcentajes de recurrencia $(58,63)$ versus visión y remoción con fórceps.

La dilatación y legrado no debe ser usado como método diagnóstico o terapéutico (35). Estudios clase II muestran una tasa de éxito en remoción de lesión de un $8-41 \%$, siendo generalmente incompleta (63). Además su utilización conlleva a dos grandes problemas: no diagnóstico de patología (pólipos pequeños) y fragmentación de pólipos más grandes (dificultades en diagnostico histopatológico) (64). Por otra parte, de requerir histeroscopia posterior, la morbilidad de la cirugía aumenta considerablemente, con hasta un $1 \%$ de perforaciones uterinas y $0,5 \%$ infecciones (1).

Quirúrgico radical. La histerectomía total es la opción quirúrgica radical y definitiva. Si bien garantiza ausencia de recurrencias y malignidad futura, es un tratamiento con potencial mayor morbilidad, invasivo y que finaliza la fertilidad, de tal forma que su indicación deberá ser justificada y en concordancia con el paciente (35). A la fecha, no existen estudios randomizados controlados que comparen la histerectomía con tratamientos conservadores (1).

\section{CONCLUSION}

La patogenia de los pólipos endometriales se encuentra actualmente en revisión, no hay ninguna teoría que explique la génesis de todos los pólipos.

El entender en profundidad la biología de un pólipo endometrial será relevante para el clínico, pues permitirá un acercamiento diagnóstico y terapéutico adecuado. Según las recomendaciones de la Sociedad Americana de Ginecólogos Laparoscopistas (AAGL) en sus reportes prácticos, la histeroscopia es el gold estándar terapéutico. El manejo conservador es aplicable a pólipos pequeños y asintomáticos, el manejo médico no puede ser recomendado a la fecha. En pacientes infértiles la resección es siempre recomendada, al igual que en pacientes sintomáticas y postmenopáusicas. Con el avance en instrumentos y técnicas endoscópicas que permiten el "see and treat" con un riesgo controlado y alta efectividad, la histeroscopia ambulatoria en la oficina se perfila como la mejor opción, pues permitirá exitosamente la extracción de pólipos menores de $2 \mathrm{~cm}$ al momento del diagnóstico, de forma sencilla y sin anestesia. Estimamos que todas las pacientes con pólipos endometriales sintomáticos 
y/o postmenopáusicos con o sin síntomas, deben ser extraídos mediante histeroscopia y resectoscopia. Siempre debe considerarse el tamaño, factores de riesgo de malignidad, condición clínica general, aspectos de fertilidad, capacidad de sus cirujanos y deseos del paciente.

\section{REFERENCIAS}

1. Salim S, Won H, Nesbitt-Hawes E, Campbell N, Abbott $\mathrm{J}$. Diagnosis and management of endometrial polyps: a critical review of the literature. J Minim Invasive Gynecol 2011;18(5):569-81.

2. Kim KR, Peng R, Ro JY, Robboy SJ. A diagnostically useful histopathologic feature of endometrial polyp: the long axis of endometrial glands arranged parallel to surface epithelium. Am J Surg Pathol 2004;28:1057-1062.

3. Vanni R, Dal Cin P, Marras S, et al. Endometrial polyp: Another benign tumor characterized by 12q13q15 changes. Cancer Genet Cytogenet 1993;68:32-3.

4. Fletcher JA, Pinkus JL, Lage JM, Morton CC, Pinkus GS. Clonal 6p21 rearrangement is restricted to the mesenchymal component of an endometrial polyp. Genes Chromosomes Cancer 1992;5:260-3.

5. Inagaki N, Ung L, Otani T, Wilkinson D, Lopata A. Uterine cavity matrix metalloproteinases and cytokines in patients with leiomyoma, adenomyosis or endometrial polyp. Eur J Obstet Gynecol Reproduct Biol 2003;111:197.203.

6. Nogueira AA, Sant'Ana de Almeida EC, Poli Neto OB, Zambelli Ramalho LN, Rosa e Silva JC, Candido dos Reis FJ. Immunohistochemical expression of p63in endometrial polyps: evidencethat a basal cell immunophenotype is maintained. Menopause 2006;13:82630.

7. Anastasiadis PG, Koutlaki NG, Skaphida PG, Galazios GC, Tsikouras PN, Liberis VA. Endometrial polyps: prevalence, detection and malignant potential in women with abnormal uterine bleeding. Eur J Gynaecol Oncol 2000;21(2):180-3.

8. Mittal K, Schwartz L, Goswami S, Demopoulos R. Estrogen and progesterone receptor expression in endometrial polyps. Int J Gynecol Pathol 1996;15(4):345-8.

9. Lopes RGC, Baracat EC, de Albuquerque Neto LC, et al. Analysis of estrogen- and progesterone-receptor expression in endometrial polyps. J Minim Invasive Gynecol 2007;14:300-3.

10. Tallini G, Vanni R, Manfioletti G, Kazmierczak B, Faa G, Pauwels P, et al. HMGI-C and HMGI(Y) immunoreactivity correlates with cytogenetic abnormalities in lipomas, pulmonary chondroid hamartomas, endometrial polyps, and uterine leiomyomas and is compatible with rearrangement of the $\mathrm{HMGl}-\mathrm{C}$ and $\mathrm{HMGI}(\mathrm{Y})$ genes. Lab Invest 2000;80:359-69.

11. Hamet P. Cancer and hypertension: a potential for crosstalk? J Hypertens 1997;15:1573-77.

12. Savelli $L$, De lacco $P$, Santini $D$, et al. Histopathologic features and risk factors for benignity, hyperplasia, and cancer in endometrial polyps. Am J Obstet Gynecol 2003;188:927-31.

13. Baiocchi G, Manci N, Pazzaglia M, Giannone L, Burnelli L, Giannone E. Malignancy in endometrial polyps: a 12-year experience. Am J Obstet Gynecol 2009;201:462.e1-462.e4.
14. Costa-Paiva Lucia, Godoy CE Jr, Antunes A Jr, Caseiro JD, Arthuso M, Aarao M. Pinto-Neto AM. Risk of malignancy in endometrial polyps in premenopausal and postmenopausal women according to clinicopathologic characteristics. Menopause 2011;18(12):1278-82.

15. Lee SC, Kaunitz AM, Sanchez-Ramos L, Rhatigan RM. The oncogenic potential of endometrial polyps: a systematic review and meta-analysis. Obstet Gynecol 2010;116(5):1197-205.

16. Gregoriou O, Konidaris S, Vranchnis N, et al. Clinical parameters linked with malignancy in endometrial polyps. Climateric 2009;12:454-58.

17. Dreisler E, Stampe Sorensen S, Ibsen PH, Lose G. Prevalence of endometrial polyps and abnormal uterine bleeding in a Danish population aged $20-74$ years. Ultrasound Obstet Gynecol 2009;33:102-108.

18. Dreisler E, Sorensen S, Lose G. Endometrial polyps and associated factors in Danish women aged 36-74 years. Am J Obstet Gynecol. 2009;200:e1-e6

19. Orvieto R, Bar-Hava I, Dicker D, Bar J, Ben-Rafael Z, Neri A. Endometrial polyps during menopause: characterization and significance. Acta Obstet Gynecol Scand 1999;78:883-6.

20. Oguz S, Sargin A, Kelekci S, Aytan H, Tapisiz OL, Mollamahmutoglu $\mathrm{L}$. The role of hormone replacement therapy in endometrial polyp formation. Maturitas 2005;50:231-6.

21. Maia H Jr, Maltez A, Studart E, Athayde C, Coutinho EM. Ki-67, Bcl-2 and p53 expression in endometrial polyps and in the normal endometrium during the menstrual cycle. Br J Obstet Gynaecol 2004;111:1242-7.

22. Kurman RJ, Mazur MT. Benign diseases of the endometrium. In: Kurman RJ, editor. Blaustein's pathology of the female genital tract. 4th ed. New York: SpringerVerlag; 1994. p.448-60

23. Coeman D, Van Belle Y, Vanderick G, De Muylder X, De Muylder E, Campo R. Hysteroscopic findings in patients with a cervical polyp. Am J Obstet Gynecol 1993;169:1563-5.

24. Vilodre LC, Bertat R, Petters R, Reis FM. Cervical polyp as risk factor for hysteroscopically diagnosed endometrial polyps. Gynecol Obstet Invest 1997;44:191195.

25. Spiewankiewicz B, Stelmachow J, Sawicki W, Cendrowski K, Kuzlik R. Hysteroscopy in cases of cervical polyps. Eur J Gynaecol Oncol 2003;24:67-9.

26. Vilodre LC, Bertat R, Petters R, Reis FM. Cervical polyp as risk factor for hysteroscopically diagnosed endometrial polyps. Gynecol Obstet Invest 1997;44:1915.

27. Clevenger-Hoeft M, Syrop CH, Stovall DW, Van Voorhis BJ. Sonohysterography in premenopausal women with and without abnormal bleeding. Obstet Gynecol 1999;94:516-520.

28. Orvieto R, Bar-Hava I, Dicker D, Bar J, Ben-Rafael Z, Neri A. Endometrial polyps during menopause: characterization and significance. Acta Obstet Gynecol Scand 1999;78(10):883-6

29. Haimov-Kochman R, Deri-Hasid R, Hamani Y, Voss E. The natural course of endometrial polyps: Could they vanish when left untreated? Fertil Steril 2009;92:828. e11-828.e12.

30. Fabres C, Alam V, Balmaceda J, Zegers-Hochschild F, Mackenna A, Fernandez E. Comparison of ultrasonography and hysteroscopy in the diagnosis of intrau- 
terine lesions in infertile women. J Am Assoc Gynecol Laparosc 1998;5:375-8.

31. Perez-Medina T, Bajo J, Huertas MA, Rubio A. Predicting atypia inside endometrial polyps. J Ultrasound Med 2002;21:125-8.

32. La Torre R, De Felice C, De Angelis C, Coacci F, Mastrone M, Cosmi EV. Transvaginal sonographic evaluation of endometrial polyps: a comparison with two dimensional and three dimensional contrast sonography. Clin Exp Obstet Gynecol 1999;26:171-3.

33. Nagele $F$, O'Connor $H$, Davies $A$, Badawy $A$, Mohamed $\mathrm{H}$, Magos A. 2500 outpatient diagnostic hysteroscopies. Obstet Gynecol 1996;88(1):87-92.

34. Van Bogaert LJ. Clinicopathologic findings in endometrial polyps. Obstet Gynecol 1988;71(5):771-3.

35. Lieng M, Istre O, Sandvik L, Qvigstad E. Prevalence, 1-year regression rate, and clinical significance of asymptomatic endometrial polyps: cross-sectional study. J Minim Invasive Gynecol-2009;16(4):465-71.

36. AAGL Practice Report: Practice Guidelines for the Diagnosis and Management of Endometrial Polyps. J Minim Invasive Gynecol 2012;19:3-10.

37. Martinez-Perez O, Perez-Medina T, Bajo-Arenas J. Ultrasonography of endometrial polyps. Ultrasound Rev Obstet Gynecol 2003;3:43-50.

38. Golan A, Sagiv R, Berar M, Ginath S, Glezerman M. Bipolar electrical energy in physiologic solution -a revolution in operative hysteroscopy. J Am Assoc Gynecol Laparosc 2001;8:252-8.

39. Hassa H, Tekin B, Senses T, Kaya M, Karatas A. Are the site, diameter, and number of endometrial polyps related with symptomatology? Am J Obstet Gynecol 2006;194:718-21.

40. Hinckley MD, Milki AA. 1000 office-based hysteroscopies prior to in vitro fertilization: feasibility and findings. JSLS 2004;8:103-7.

41. Shokeir TA, Shalan HM, EI-Shafei MM. Significance of endometrial polyps detected hysteroscopically in eumenorrheic infertile women. J Obstet Gynaecol Res 2004;30:84-9.

42. Perez-Medina T, Bajo-Arenas J, Salazar F, et al. Endometrial polyps and their implication in the pregnancy rates of patients undergoing intrauterine insemination: a prospective, randomized study. Hum Reprod 2005;20:1632-5.

43. Martinez MA, Jou P, Novell R, Cardona M. Endometrial polyps: risk of transformation and clinico-anatomic correlation. Prog Obstet Ginecol 2004;11:506-10.

44. Antunes A Jr, Costa-Paiva L, Arthuso M, Costa JV, Pinto-Neto AM. Endometrial polyps in pre- and postmenopausal women: factors associated with malignancy. Maturitas 2007;57:415-21.

45. Lieng M, Istre O, Qvigstad E. Treatment of endometrial polyps: a systematic review. Acta Obstet Gynecol Scand 2010;89:992-1002.

46. Ben-Arie A, Goldchmit C, Laviv Y, et al. The malignant potential of endometrial polyps. Eur J Obstet Gynecol Reprod Biol 2004;115:206-10.

47. Ferrazzi E, Zupi E, Leone FP, et al. How often are endometrial polyps malignant in asymptomatic postmenopausal women? A multicenter study. Am J Obstet Gynecol 2009;200:235.e1-235.e6.

48. Papadia A, Gerbaldo D, Fulcheri E, et al. The risk of premalignant and malignant pathology in endometrial polyps: should every polyp be resected? Minerva Ginecol 2007;59:117-24.

49. Ferrazzi E, Zupi E, Leone FP, Savelli L, Omodei U, Moscarini $\mathrm{M}$, et al. How often are endometrial polyps malignant in asymptomatic postmenopausal women? A multicenter study. Am J Obstet Gynecol 2009;200(3):235.e1-6.

50. Lenci MA, Lui do Nascimento VA, Grandini AB, et al. Premalignant and malignant lesions in endometrial polyps in patients undergoing hysteroscopic polypectomy. Einstein (Sao Paulo) 2014;12(1):16-21.

51. Silva Reis PA, Nogueira AA, Reis FJ, Campolungo A, Carrara $\mathrm{HH}$, Andrade JM. Is hysteroscopic appearance a safe means of differentiating malignant from benign endometrial polyps in postmenopausal women? Gynaecol Endosc 2001;10(1):49-51.

52. Trimble CL, Kauderer J, Zaino R, et al. Concurrent endometrial carcinoma in women with a biopsy diagnosis of atypical endometrial hyperplasia: a Gynecologic Oncology Group study. Cancer 2006;106:812-9.

53. Ben-Arie A, Goldchmit C, Laviv Y, Levy R, Caspi B, Huszar $\mathrm{M}$, et al. The malignant potential of endometrial polyps. Eur J Obstet Gynecol Reprod Biol 2004;115:206-10.

54. DeWaay DJ, Syrop CH, Nygaard IE, Davis WA, Van Voorhis BJ. Natural history of uterine polyps and leiomyomata. Obstet Gynecol 2002;100:3-7.

55. Spiewankiewicz B, Stelmachow J, Sawicki W, Cendrowski K, Wypych P, Swiderska K. The effectiveness of hysteroscopic polypectomy in cases of female infertility. Clin Exp Obstet Gynecol 2003;30: 23-5.

56. Perez-Medina T, Bajo J, Huertas MA, Rubio A. Predicting atypia inside endometrial polyps. J Ultrasound Med 2002;21(2):125-8.

57. Vercellini P, Trespidi L, Bramante T, Panazza S, Mauro F, Crosignani PG. Gonadotropin releasing hormone agonist treatment before hysteroscopic endometrial resection. Int J Gynecol Obstet 1994;45:235-9.

58. Preutthipan S, Herabutya Y. Hysteroscopic polypectomy in 240 premenopausal and postmenopausal women. Fertil Steril 2005;83: 7059.

59. Emanuel $\mathrm{MH}$, Wamsteker $\mathrm{K}$. The intra uterine morcellator: a new hysteroscopic operating technique to remove intrauterine polyps and myomas. J Minim Invasive Gynecol 2005;12:62-6.

60. Jansen FW, Vredevoogd CB, Van Ulzen K, Hermans $\mathrm{J}$, Trimbos JB, Trimbos-Kemper TCM. Complications of hysteroscopy: a prospective, multicenter study. Obstet Gynecol 2000;96:266-70.

61. Nathani F, Clark TJ. Uterine polypectomy in the management of abnormal uterine bleeding: a systematic review. J Minim Invasive Gynecol 2006;13:260-8.

62. Lieng M, Istre O, Sandvik L, Engh V, Qvigstad E. Clinical effectiveness of transcervical polyp resection in women with endometrial polyps: randomized controlled trial. J Minim Invasive Gynecol 2010;17:351-7.

63. Salim S, Won H, Nesbitt-Hawes E, Campbell N, Abbott $\mathrm{J}$. Diagnosis and management of endometrial polyps: a critical review of the literature. J Minim Invasive Gynecol 2011;18(5):569-81.

64. Bettocchi S, Ceci O, Vicino M, Marello F, Impedovo L, Selvaggi L. Diagnostic inadequacy of dilatation and curettage. Fertil Steril 2001;75:803-5. 